\title{
THE F1-F2 VOWEL CHART FOR CZECH WHISPERED VOWELS A, E, I, O, U
}

\author{
Michal Grepla ${ }^{\mathrm{a}}$, Tomas Furst ${ }^{\mathrm{b}}$, Josef Pesak ${ }^{\mathrm{a}}$ \\ ${ }^{a}$ Department of Medical Biophysics, Faculty of Medicine, Palacky University, Olomouc, Czech Republic \\ ${ }^{b}$ Department of Mathematical analysis and Application of Mathematics, Faculty of Science, Palacky University, \\ Olomouc. \\ e-mail: greplm@fnol.cz
}

Received: August 20, 2007; September 13, 2007

Keywords: Formant frequencies/Formant chart/Spectral analysis/Vowel/Czech

Aim: The aim of this contribution is to present the formant chart of the Czech vowels a, e, i, o, u and show that this can be achieved by means of digital methods of sound processing.

Method: A group of 35 Czech students of the Pedagogical Faculty of Palacky University was tested and a record of whispered vowels was taken from each of them. The record was digitalized and processed by the Discrete Fourier Trasform. The result is the power spectrum of the individual vocals - the graphic output consists of a plot of the relative power of individual frequencies in the original sound. The values of the first two maxima which represent the first and the second formants were determined from the graph. The values were plotted on a formant chart.

Results: Altogether, 175 spectral analyses of individual vowels were performed. In the resulting power spectrum, the first and the second formant frequencies were identified. The first formant was plotted against the second one and pure vocal formant regions were identified.

Conclusion: Frequency bands for the Czech vowel "a" were circumscribed between 850 and $1150 \mathrm{~Hz}$ for first formant (F1) and between 1200 and $2000 \mathrm{~Hz}$ for second formant (F2). Similarly, borders of frequency band for vowel "e" they were 700 and $950 \mathrm{~Hz}$ for F1 and 1700 and $3000 \mathrm{~Hz}$ for F2. For vowel "i" 300 and $450 \mathrm{~Hz}$ for F1 and 2000 and $3600 \mathrm{~Hz}$ for F2, for vowel "o" 600 and $800 \mathrm{~Hz}$ for F1 and 600 and $1400 \mathrm{~Hz}$ for F2, for vowel "u" $100 \mathrm{and} 400 \mathrm{~Hz}$ for $\mathrm{F} 1$ and 400 and $1200 \mathrm{~Hz}$ for F2.

Discussion: At low frequencies it is feasible to invoke the source-filter model of voice production and associate vowel identity with frequencies of the first two formants in the voice spectrum. On the other hand, subject to intonation, singing or other forms of exposed voice (such as emotional speech, focused speech), the formant regions tend to spread. In spectral analysis other frequencies dominate, so specific formant frequency bands are not easily recognizable.

Although the resulting formant map is not much different from the formant map of Peterson, it carries basic information about specific Czech vowels. The results may be used in further research and in education.

\section{INTRODUCTION}

Like music, speech consists of groups of times, these groups are made of separate times, and times are formed by syllables. Syllables contain speech sounds - consonants and vowels. Consonants are characterized by pressure disturbances originating from constriction or closure at one or more points along the vocal tract. In contrast to consonants, vowels share more similarities with tones.

Vowels are perceived and classified on the basis of the two lowest formant frequencies (F1 and F2) of the vocal tract $^{1,2}$. A complex tone of many harmonics, generated by vocal fold vibrations of the larynx, is acoustically filtered by the vocal tract according to these formant frequencies ${ }^{3}$. The vocal tract acts as a resonant cavity, as the position of the jaw, lips, and tongue affect the parameters of the resonant cavity, resulting in different formant values.

Any acoustic signal (including speech sounds) is a pressure wave, which can theoretically be represented as a continuous function of time. A digital sound record consists of a vector the components of which represent rounded values of pressure (at the position of the record- er) at successive instants. Such a vector can be processed by Discrete Fourier Transform (DFT) - a digital version of continuous Fourier analysis. The magnitude of the resulting vector carries information about the relative proportion of individual frequencies in the original sound signal.

Fourier analysis is based on the observation that any periodic signal can be decomposed into a weighed sum of individual perfect tones, without any loss of information. By a perfect tone we understand a periodic signal of an ideal sinusoid form (i.e. a signal the spectrum of which consists of a single frequency).

\section{MATERIAL AND METHODS}

A group of 35 Czech students of the Pedagogical Faculty of Palacky University was tested (21 male, 14 female, aged 21-28 years, average age 23.15). A record of all the Czech vocals - a, e, i, o, u - was taken from each person. The file contained vocals whispered in a modal voice. The vocals were whispered so that the fundamen- 

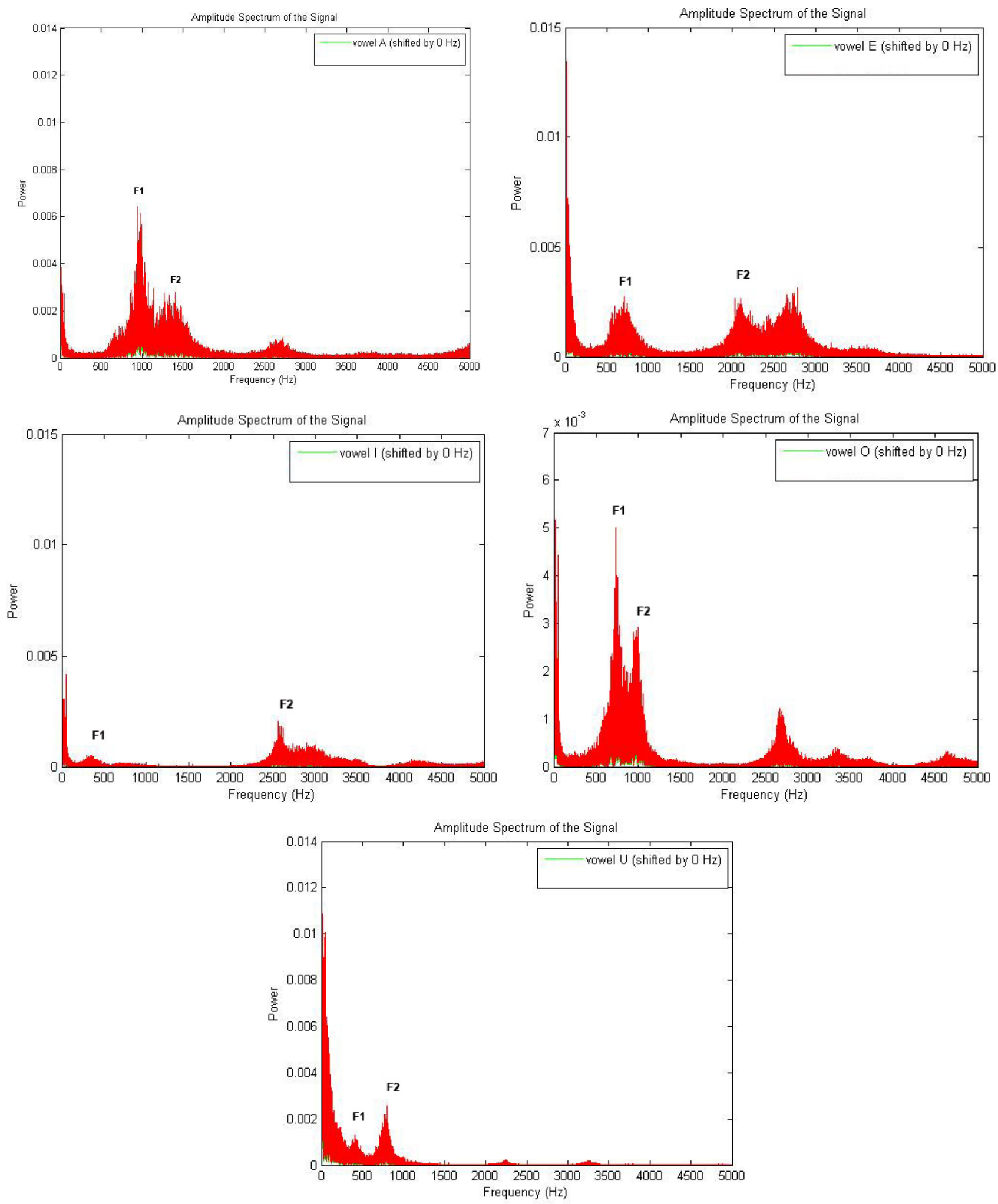

Fig. 1. Spectral analysis of the individual vocals a, e, i, o, u.

tal frequency of the full (non-whispered) voice would not mask the formants. The record was digitalized by means of the Sound Forge software $(44.1 \mathrm{kHz}$ sampling frequency, 16-bit mono track) and cut into separate sequences, each containing a single vocal of one second in duration. The record was further processed by the Fast
Fourier Transform (FFT) by means of the mathematical software MatLab. The result is the power spectrum of the individual vocals - the graphic output consists of a plot of the relative power of individual frequencies in the original sound. The values of the first two maxima were determined from the graph. These maxima represent the 


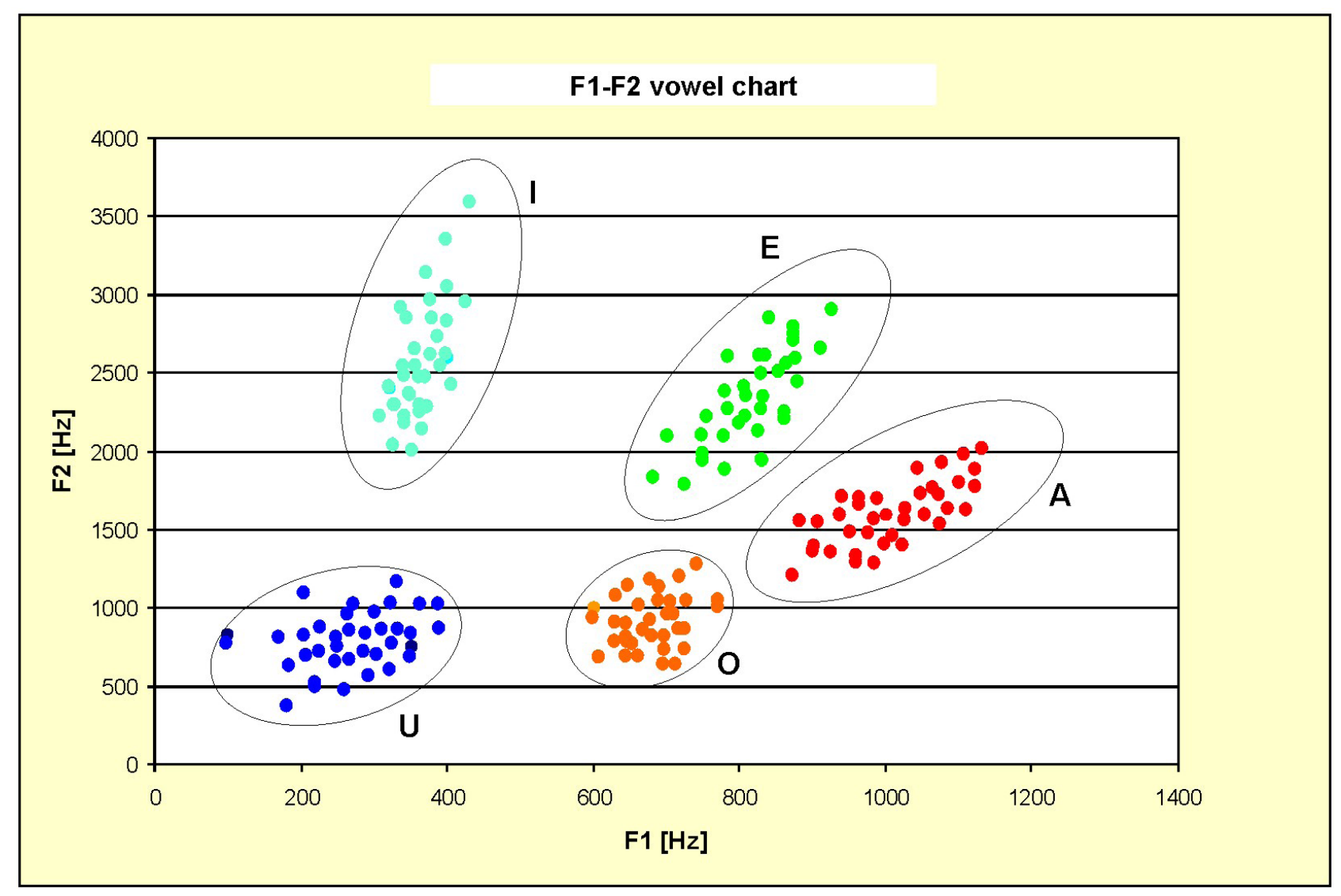

Fig. 2. Formant map of the two lowest formants of whispered Czech vocals.

first and the second formants. The values were plotted on a formant chart.

\section{RESULTS}

Altogether, 175 spectral analyses were performed. For clarity of results, whispered vocals are preferred because they do not contain the fundamental frequency (and the overtones) of the carrier-tone produced by the vocal folds. These frequencies would otherwise dominate the power spectrum and effectively mask the formant regions. In the case of whispered vocals, the formant regions are clearly visible. In Figure 1 spectral analyses of the individual vocals are depicted. The first two maxima can clearly be identified. The peak at $50 \mathrm{~Hz}$ is present in all the vocals - it is an artifact produced by the recording technique. The frequency of the first formant was plotted against the frequency of the second one and pure vocal formant regions were identified (Fig. 2).

\section{CONCLUSION}

Frequency bands for the Czech vowel "a" were circumscribed between 850 and $1150 \mathrm{~Hz}$ for first formant (F1) and between 1200 and $2000 \mathrm{~Hz}$ for second formant (F2). Similarly, borders of frequency band for vowel "e" they were 700 and $950 \mathrm{~Hz}$ for $\mathrm{F} 1$ and 1700 and $3000 \mathrm{~Hz}$ for F2. For vowel "i” 300 and $450 \mathrm{~Hz}$ for F1 and 2000 and $3600 \mathrm{~Hz}$ for F2, for vowel "o" 600 and $800 \mathrm{~Hz}$ for F1 and 600 and $1400 \mathrm{~Hz}$ for F2, for vowel "u" 100 and $400 \mathrm{~Hz}$ for F1 and 400 and $1200 \mathrm{~Hz}$ for F2.

As shown in Figure 2 each formant occupies a welldefined region. The regions are relatively compact. This can be explained by the selection of probands and by the choice of modal and whispered voice.

The position of the formants corresponds closely to the map of the Czech vocals in the Helwag triangle ${ }^{4}$.

\section{DISCUSSION}

Vowel identity is somewhat problematic because of its dependence on fundamental frequency. At low frequencies it is feasible to invoke the source-filter model of voice production and associate vowel identity with frequencies of the first two formants in the voice spectrum. Displayed on two dimensional picture - the vowel chart - the position of formants approximates the articulary space, with the F1 dimension correlating with jaw opening and the F2 dimesion with front or back placement of the tongue. The simplified graphic expression is shaped like a triangle with "u", "a" and "i" as its vertices. This phenomenon was described by Helwag in 1781, but then only in relation to the position of the tongue in mouth cavity. 
On the other hand, subject to intonation, singing or other forms of exposed voice (such as emotional speech, focused speech), the formant regions tend to $\operatorname{spread}^{5-8}$. In spectral analysis other frequencies dominate, so specific formant frequency bands are not easily recognizable.

Although the resulting formant map is not much different from the formant map of Peterson ${ }^{1}$, it carries basic information about specific Czech vowels. The results may be used in further research and in education. Furthermore, this knowledge applied together with real-time spectral analyzer can be used as a biofeedback method for practicing vowel pronunciation for singing pedagogy or for patients after brain stroke.

\section{ACKNOWLEDGEMENTS}

This work was supported by the Council of Czech Government (MSM 6198959214).

This work was prepared with the support of university association GSVS and equipment acquired from CEZ:J14/98 and MSM152100018 of Czech Republic.

\section{REFERENCES}

1. Peterson GE, Barney HL. Control methods used in a study of vowels. Journal of the Acoustical Society of America. 1952; 24:175184.

2. Bogert BP, Peterson GE. (1957). The acoustics of speech. In L. E. Travis (Ed.), Handbook of speech pathology. 1957; p.109-173.

3. Titze IR. Priciples of voice production, Prentice Hall; 1994; 1st edition (March 14), Englewood Cliffs, New Jersey, ISBN-10: 013717893X

4. Modrackova L, Grepl M, Laichman S, Pesak J. K lidské řeči (About human speech). Collection of lectures of 14th workshop of university association for voice and speech study (GSVS). Olomouc; December 2005, p.21-26.

5. Miller R. English, French, German, and Italian techniques of singing: A study in national tonal preferences and how they relate to functional efficiency. Metuchen, NJ: Scarecrow Press; 1977.

6. Sundberg J. Acoustics of singing voice, Scientific American. 1997; 236:82-91.

7. Zhenglai Gu, Hiroki Mori, Hideki Kasuya. Analysis of vowel formant frequency variations between focus and neutral speech in Mandarin Chinese, Acoustical Science and Technology. 2003; 4(4):192-193.

8. Kienast M, SendlmeierW. Acoustical analysis of spectral and temporal changes in emotional speech, ISCA Workshop on Speech and Emotion, Northern Ireland, 2000. 